International Mathematical Forum, 2, 2007, no. 60, 2983 - 3000

\title{
A New Method for Solving First-Order Constant Coefficient Systems of Linear Differential Equations
}

\author{
Zhang Xiao-yong ${ }^{1}$ \\ Department of Mathematics \\ Shanghai Maritime University \\ Pudong Road 1550, Shanghai 200135, China \\ Sui Jiang Hua \\ Department of Mathematics \\ Dalian Maritime University \\ Dalian 116026, China
}

\begin{abstract}
In this paper, we introduce a new method for solving first-order systems of linear differential equations with constant coefficient.
\end{abstract}

Keywords: A new method, The systems of linear differential equations

\section{Introduction}

We know that many problems in science and engineering are described by differential equations. So solving various differential equations exactly have important significance in people'science action. When Newton proposed calculus, differential equation as a branch of mathematics came into being. Through hard work for several hundred years, people have been could solve many kinds of differential equations. For example, first-order homogeneous linear differential equation and first-order nonhomogeneous linear differential equation,Euler equation, higher-order constant coefficient linear differential equation and so on. But,practice for many years demonstrate that now it is very difficult that people find a kind of method for solving some sort of differential equations

\footnotetext{
1e-mail: zhxiyo@gmail.com
} 
again. Even so, solving differential equation exactly is important course in mathematics investigation yet.

In this paper, we propose a new method for solving the following first-order constant coefficient linear differential equations:

$$
\left\{\begin{array}{l}
\frac{d x}{d t}=A x \\
x(0)=\eta
\end{array}\right.
$$

Calculation demonstrate that the method is correct.

\section{Preliminaries}

In this section,we first discuss some knowledge about linear algebra.

We first introduce some notation. Suppose that $A$ is $n \times n$ constant matrix, $\lambda_{1}, \lambda_{2}, \cdots, \lambda_{k}$ are $\mathrm{k}$ distinct eigenvalues of $A$, their multiplicities are $n_{1}, n_{2}, \cdots, n_{k}$ respectively. Appreciably

$$
n_{1}+n_{2}+\cdots+n_{k}=n
$$

For every eigenvalue $\lambda_{j}$ multiplicity of which is $n_{j}$, we denote by $U_{j}$ the set of solutions of the following linear equations:

$$
\left(A-\lambda_{j} E\right)^{n_{j}} u=0
$$

Lemma 2.1 Assume $n_{2}=n_{1}+r, 0 \leq r<n_{1}, n=n_{1}+n_{2}$ and

$$
\begin{aligned}
& E_{n_{1} \times n_{1}}^{1}=\left(\begin{array}{ccccc}
\lambda_{1} & 1 & & & \\
& \lambda_{1} & 1 & & \\
& \ddots & & \\
& & \lambda_{1} & 1 \\
& & & \lambda_{1}
\end{array}\right) \quad E_{n_{2} \times n_{2}}^{2}=\left(\begin{array}{rrrr}
\lambda_{2} & 1 & & \\
& \lambda_{2} & 1 & \\
& & \ddots & \\
& & \lambda_{2} & 1 \\
& & & \lambda_{2}
\end{array}\right)_{n_{2} \times n_{2}} \\
& A=\left(\begin{array}{cc}
E^{1} & 0 \\
0 & E^{2}
\end{array}\right)_{n \times n} \quad B=\left(\begin{array}{cc}
E^{2} & 0 \\
0 & E^{1}
\end{array}\right)_{n \times n}
\end{aligned}
$$

Then there exists invertible matrix $C$ such that $C A C^{-}=B$. 


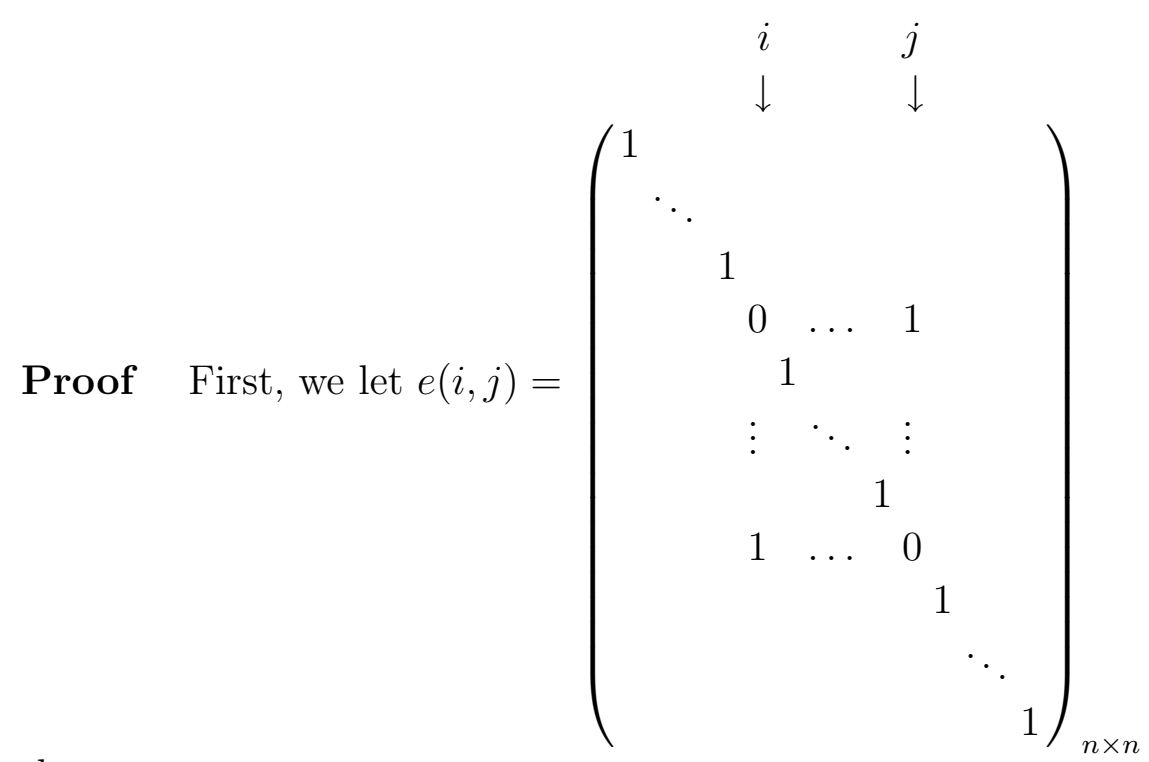

then

$$
e(i, j) \times e(i, j)=E
$$

That is to say

$$
e^{\dashv}(i, j)=e(i, j)
$$

Commuting row 1 with row $n_{1}+1$, row 2 with row $n_{1}+2 \cdots$ row $n_{1}$ with row $2 n_{1}$, matrix $A$ change into

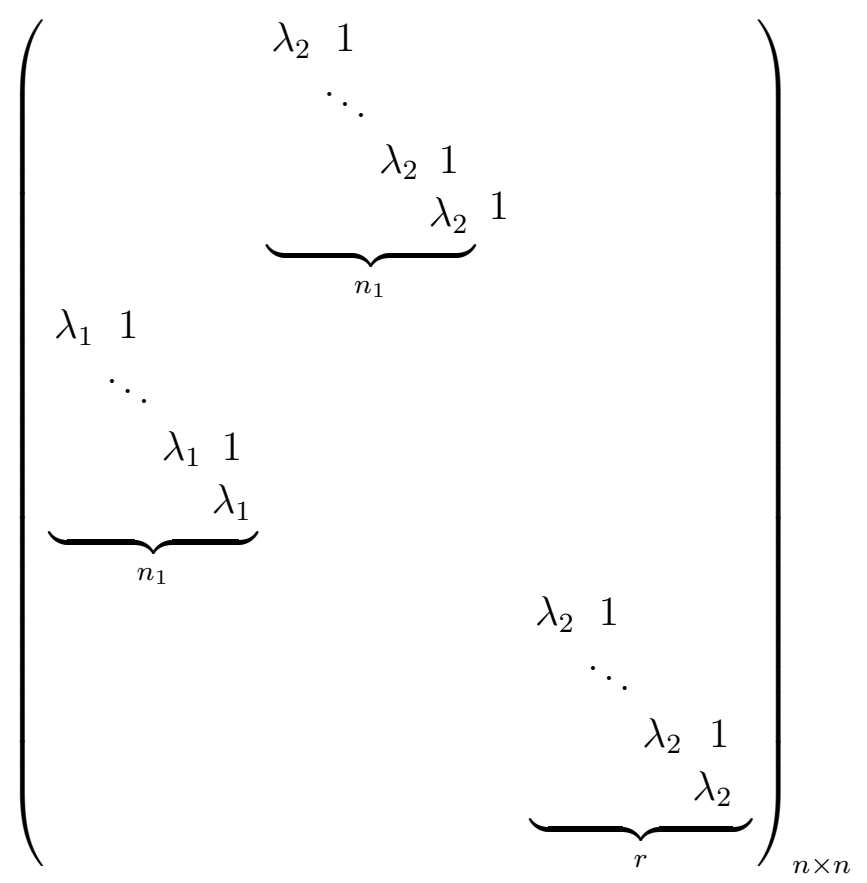


Commuting column 1 with column $n_{1}+1$,commuting column 2 with column $n_{1}+2$ correspondingly until column $n_{1}$ with column $2 n_{1}$, which leads to that

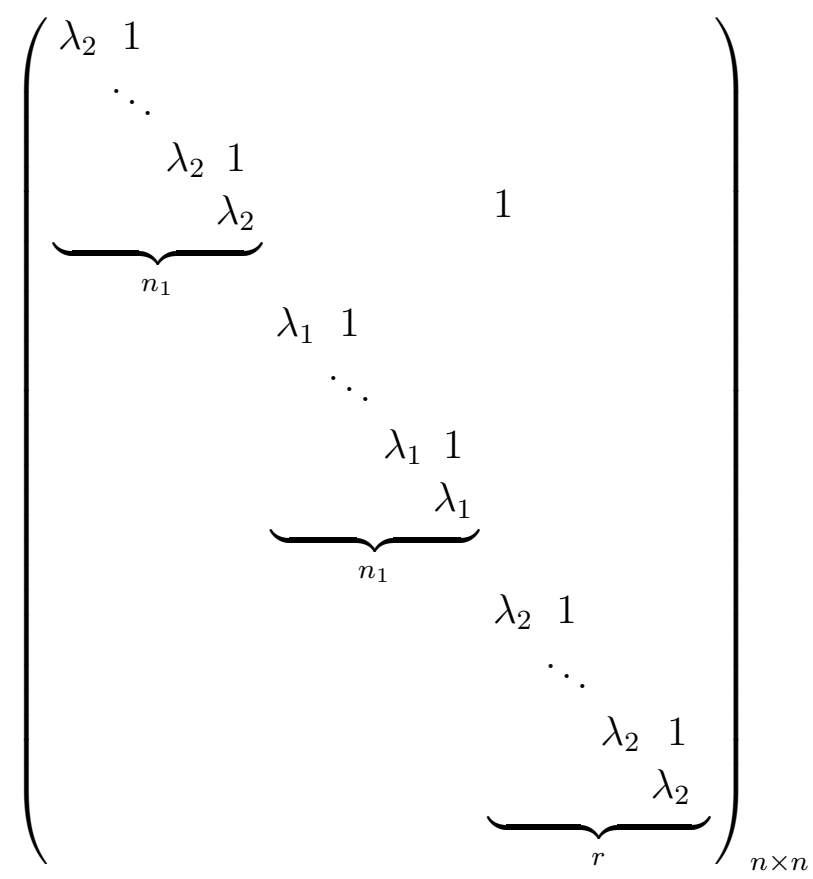

Let $C_{1}=e\left(n_{1}, 2 n_{1}\right) e\left(n_{1}-1,2 n_{1}-1\right) \ldots e\left(1, n_{1}+1\right)$ and $C_{1}^{-\dashv}=e\left(1, n_{1}+\right.$ $1) \ldots e\left(n_{1}-1,2 n_{1}-1\right) e\left(n_{1}, 2 n_{1}\right)$, we can write above matrix as

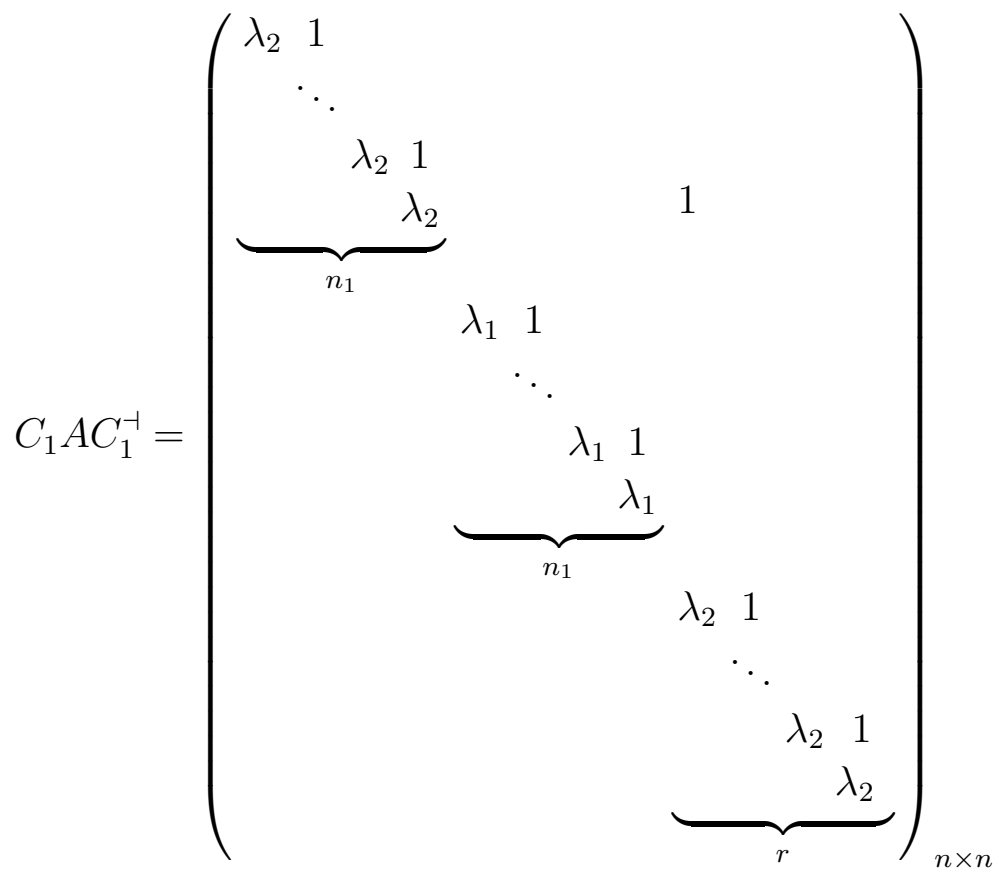

Commuting row $n_{1}+1$ with row $2 n_{1}+1$, row $n_{1}+2$ with row $2 n_{1}+2 \cdots$ row $n_{1}+r$ with row $2 n_{1}+r$, and commuting column $n_{1}+1$ with column $2 n_{1}+1$, column $n_{1}+2$ with column $2 n_{1}+2 \cdots$ column $n_{1}+r$ with column $2 n_{1}+r$ 
correspondingly, we have that

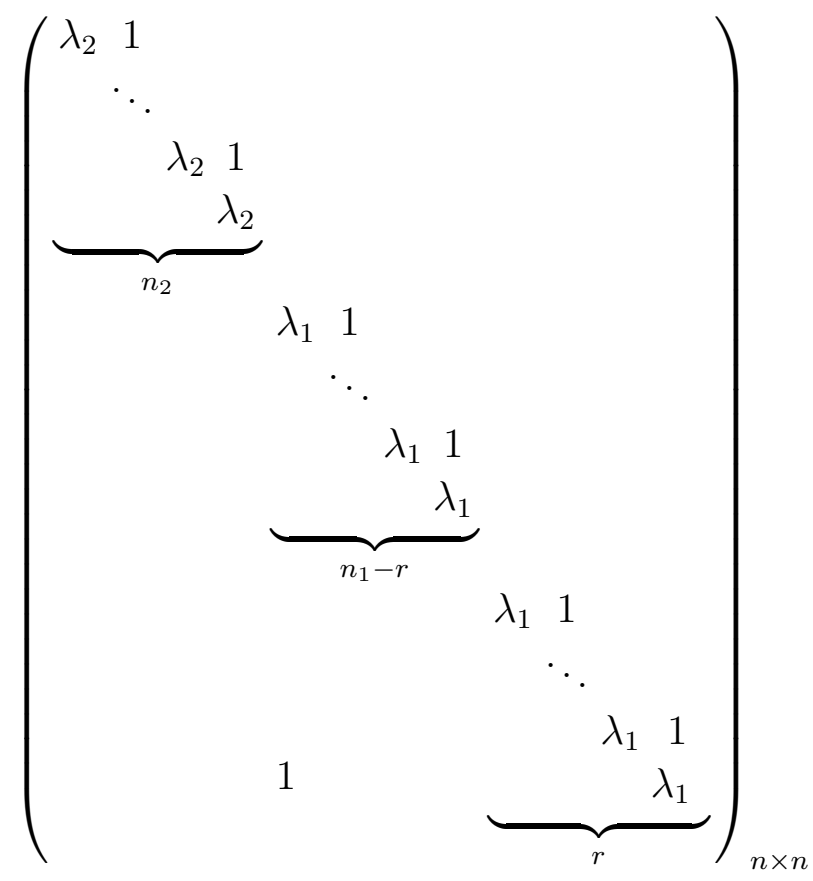

Let $C_{2}=e\left(n_{1}+r, 2 n_{1}+r\right) e\left(n_{1}+r-1,2 n_{1}+r-1\right) \ldots e\left(n_{1}+1,2 n_{1}+1\right)$, then

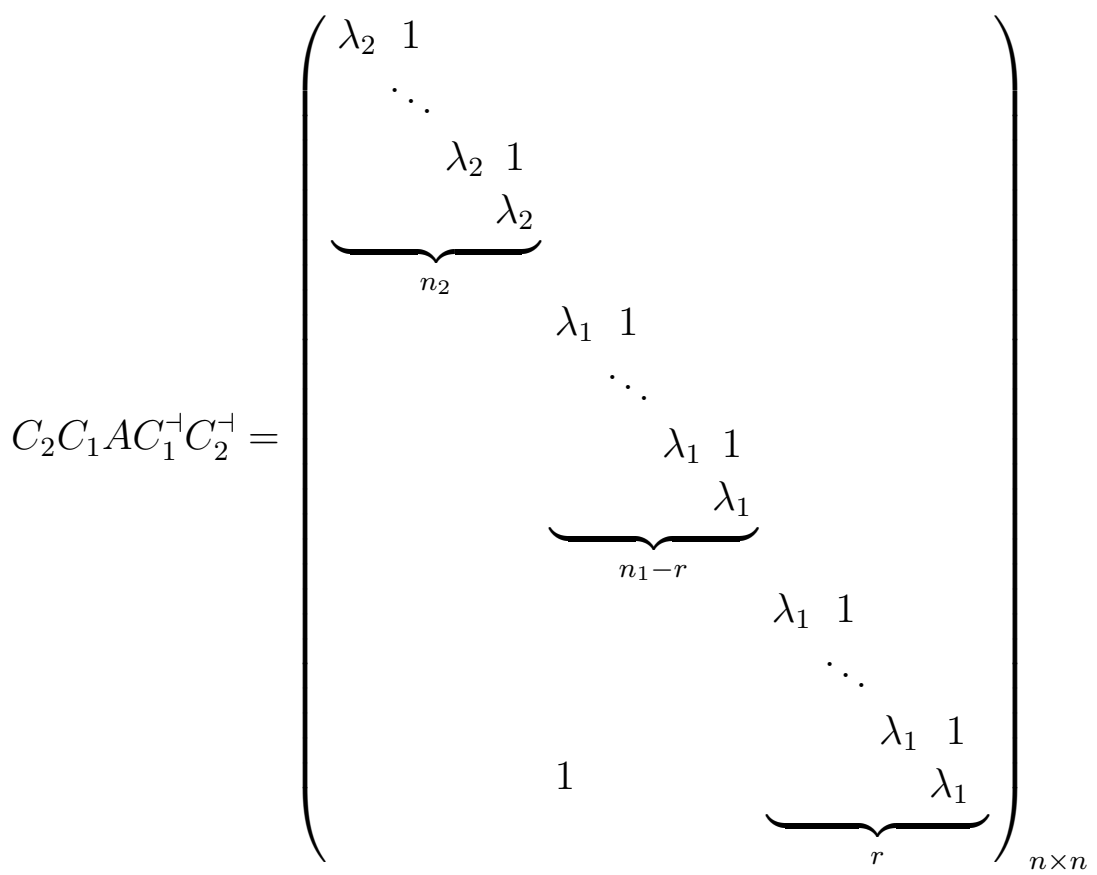


Commuting row $n$ with row $n-1$, row $n-1$ with row $n-2$ until row $n_{2}+2$ with row $n_{2}+1$, which leads to that

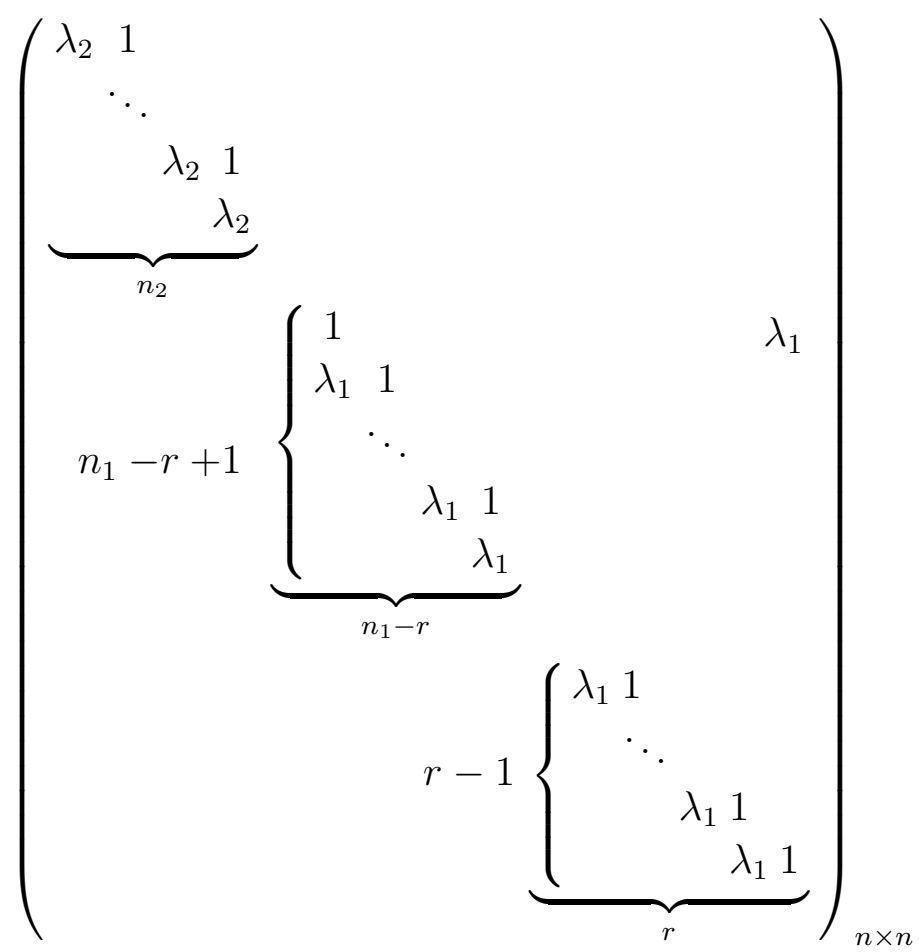

Commuting column $n$ with column $n-1$, column $n-1$ with column $n-2$ correspondingly until column $n_{2}+2$ with column $n_{2}+1$, we get that

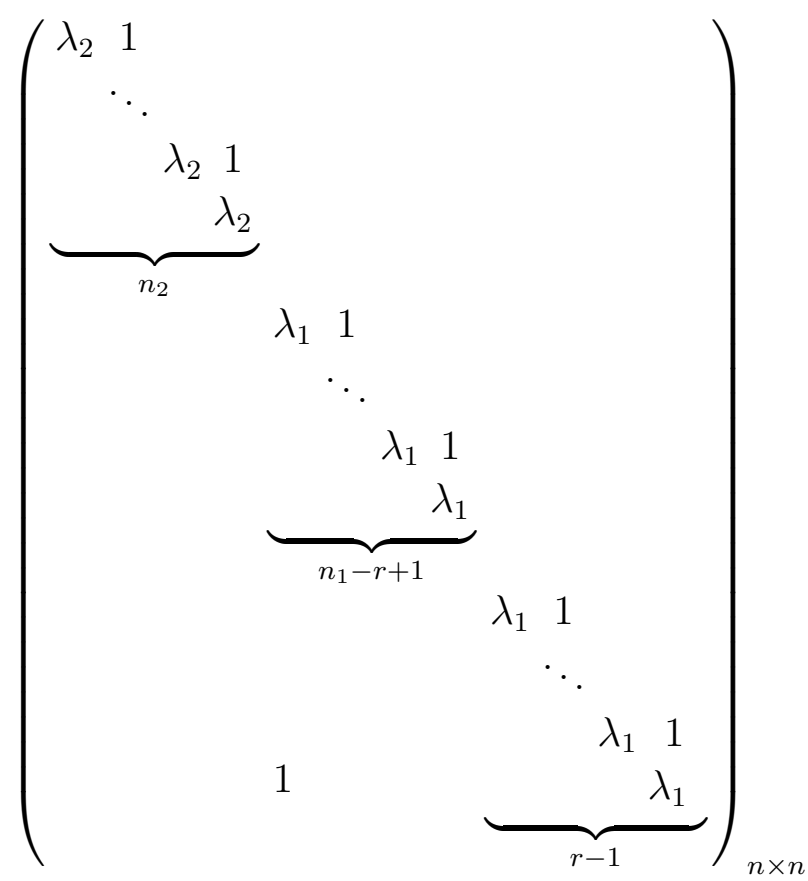


That is to say

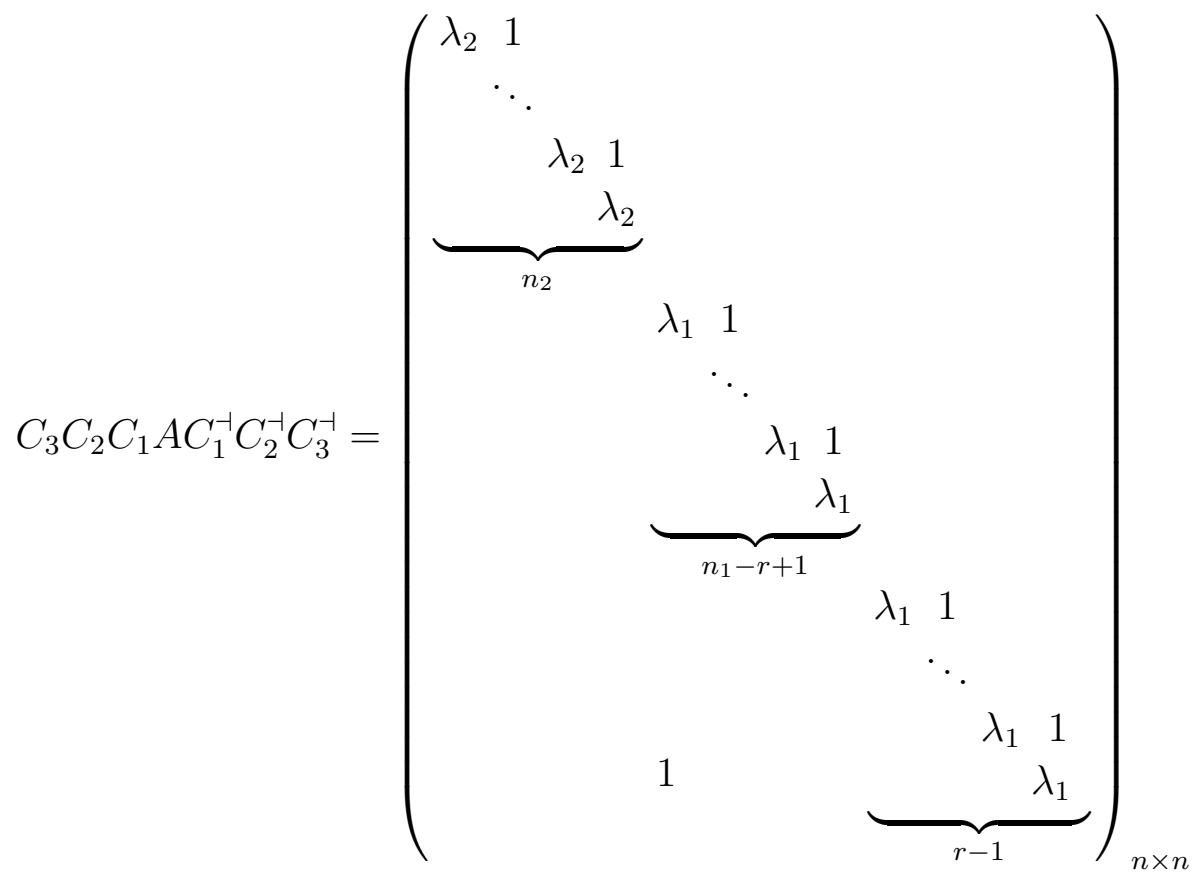

here $C_{3}=e\left(n_{2}+1, n_{2}+2\right) e\left(n_{2}+2, n_{2}+3\right) \ldots e(n-1, n)$.

Repeating about two step,we obtain that

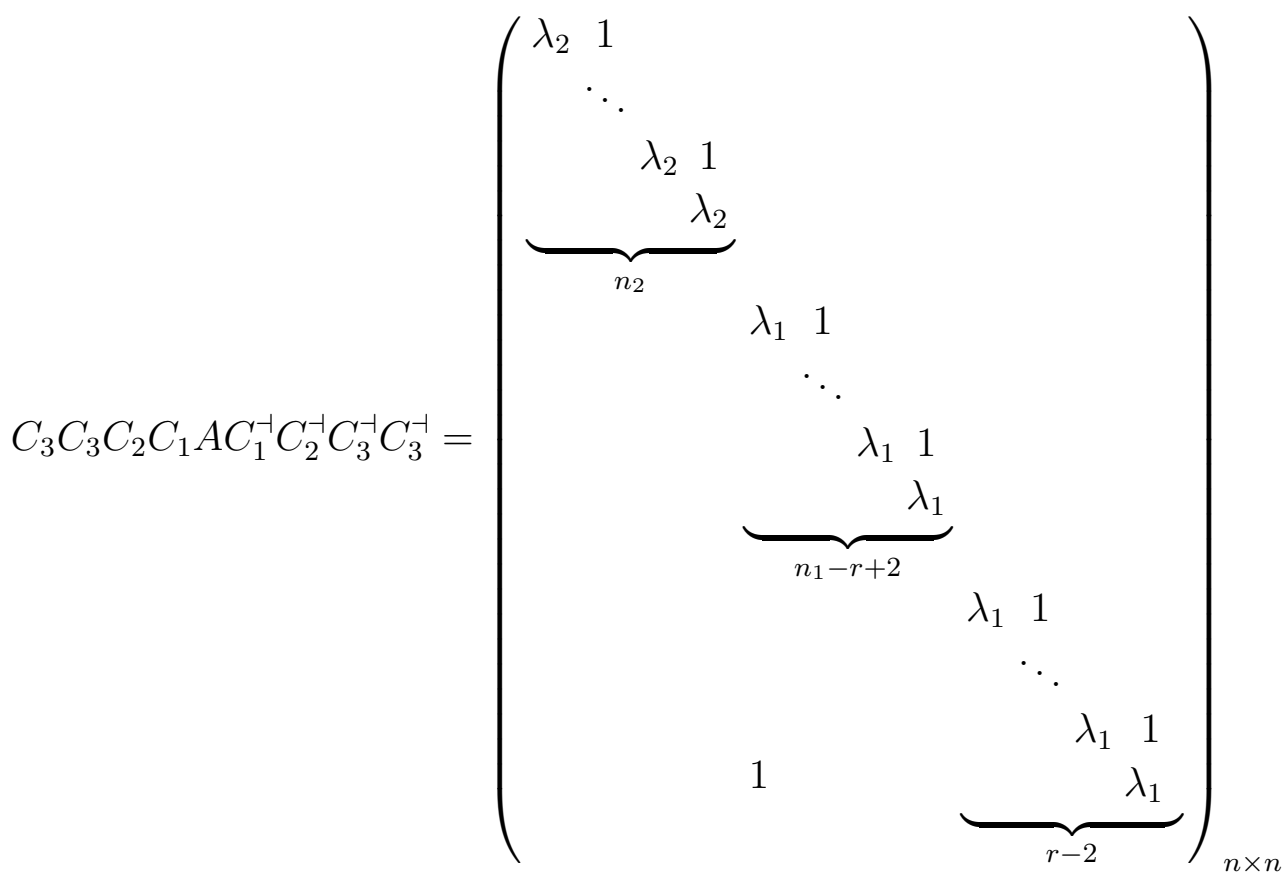


Repeating about step $r$ times, we reach the following result

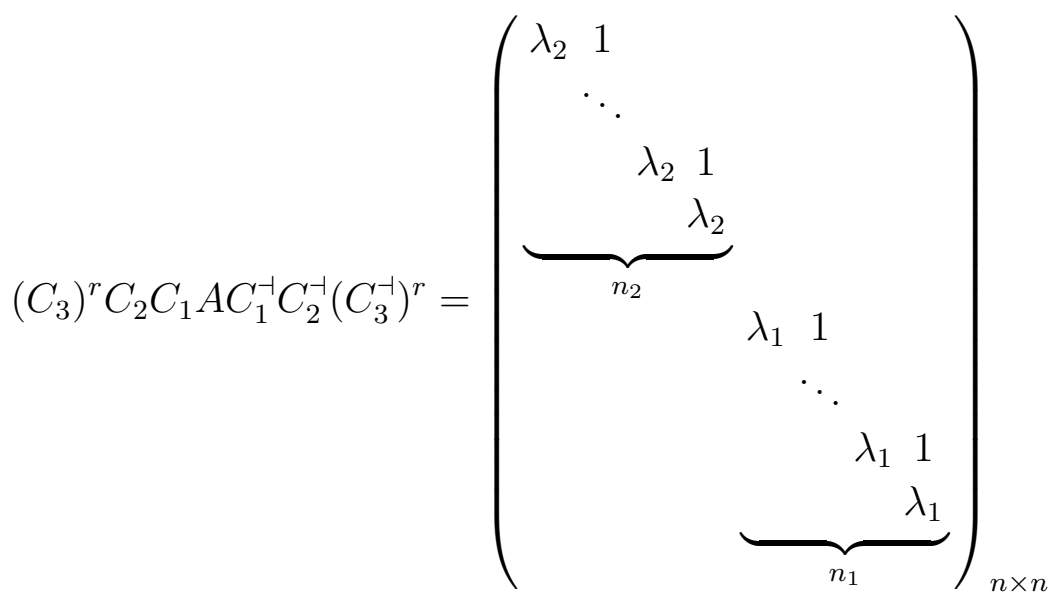

This is the desired result. Similarly,we can prove the case $n_{2}=k \times n_{1}+r$ and the case $n_{1}>n_{2}$.

Theorem 2.1 Assume that $v_{1}^{j}, v_{2}^{j}, \cdots, v_{n_{j}}^{j}(j=1,2, \cdots, k)$ is a basis of solution space of linear equations $(2.1)$ with $\lambda_{j}$, then

$$
v_{1}^{1}, v_{2}^{1}, \cdots, v_{n_{1}}^{1}, v_{1}^{2}, v_{2}^{2}, \cdots, v_{n_{2}}^{2}, \cdots, v_{1}^{k}, v_{2}^{k}, \cdots, v_{n_{k}}^{k}
$$

is a basis of $\mathrm{n}$ dimension vector space $U$.

Proof By the Jordon normal form theorem and lemma 2.1, there exists invertible matrix $B$ such that

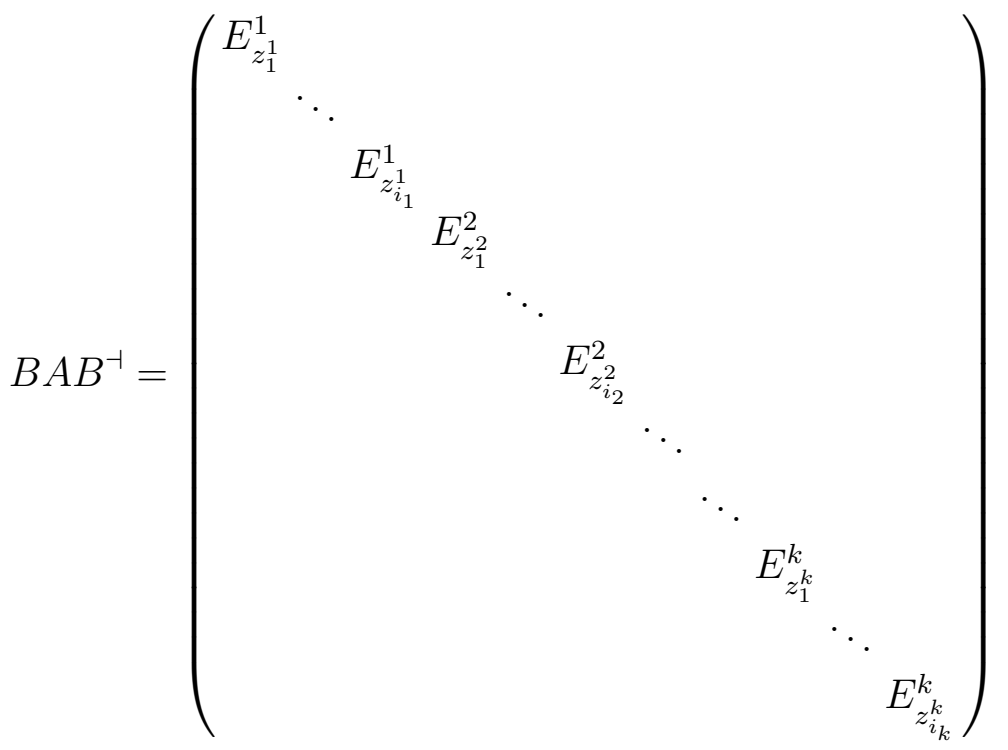

Where $z_{l_{j}}^{j}\left(l_{j}=1,2, \cdots, i_{j}\right)$ are positive integer which satisfy that

$$
z_{1}^{j}+z_{2}^{j}+\cdots+z_{i_{j}}^{j}=n_{j} \quad(j=1,2, \cdots, k)
$$




$$
E_{z_{l_{j}}^{j}}^{j}=\left(\begin{array}{ccccc}
\lambda_{j} & 1 & & & \\
& \lambda_{j} & 1 & & \\
& & \ddots & \\
& & \lambda_{j} & 1 \\
& & & \lambda_{j}
\end{array}\right)_{z_{l_{j}}^{j} \times z_{l_{j}}^{j}}
$$

Multiplying (2.1) from left side by $B$, we have that

$$
\left[B\left(A-\lambda_{j} E\right) B^{\dashv}\right]^{n_{j}}[B u]=0
$$

Taking $\lambda_{j}=\lambda_{1} \operatorname{in}(2.4)$ and Let $B u=u^{*},(2.4)$ is changed into
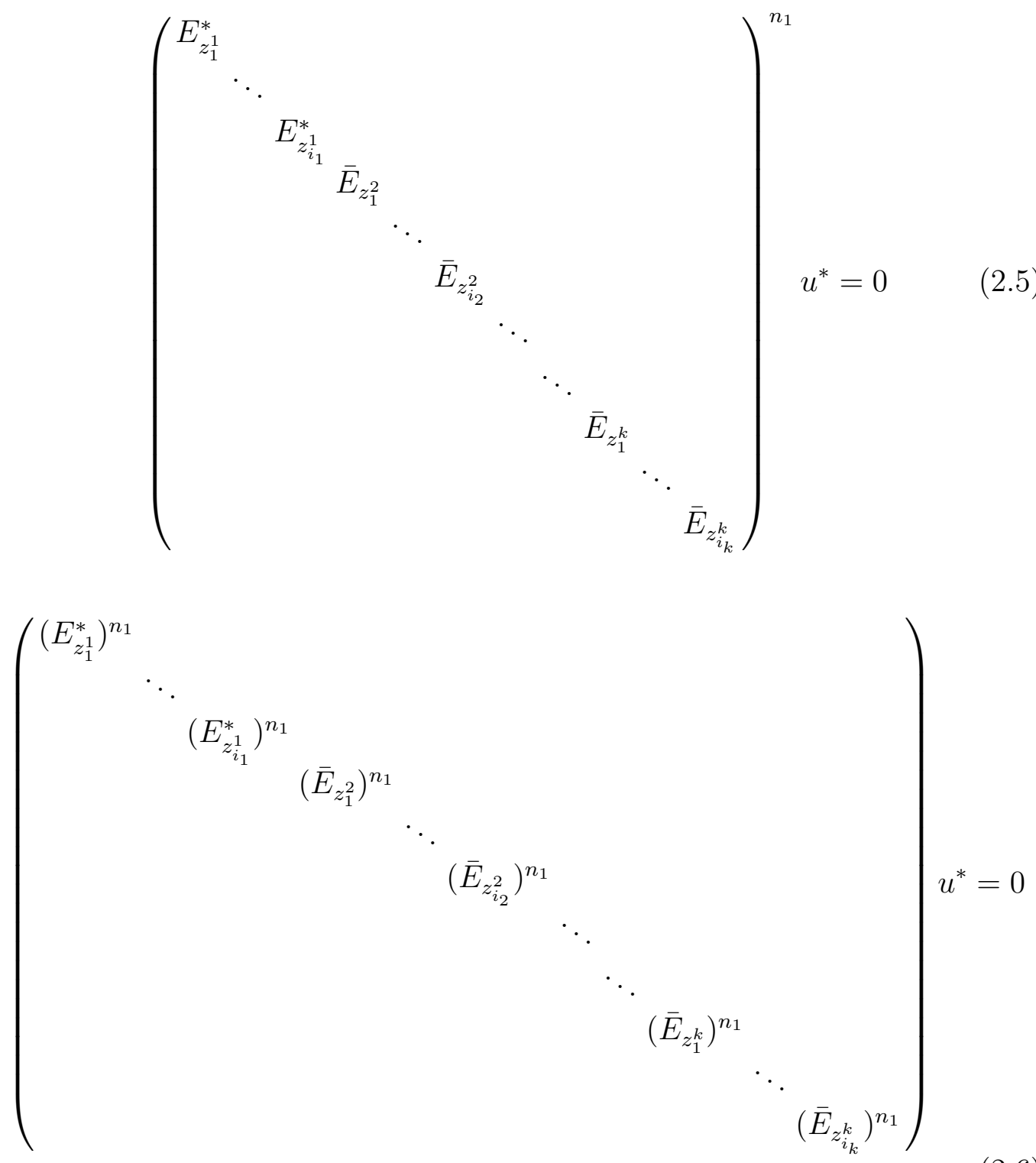
where

$$
\begin{aligned}
& E_{z_{l_{1}}^{1}}^{*}=\left(\begin{array}{rrrr}
0 & 1 & & \\
& 0 & 1 & \\
& \ddots & & \\
& & 0 & 1 \\
& & & 0
\end{array}\right) \quad\left(l_{1}=1,2, \cdots, i_{1}\right) \\
& \bar{E}_{z_{l_{j}}}=\left(\begin{array}{rrrr}
\left(\lambda_{j}-\lambda_{1}\right) & 1 & & \\
& \left(\lambda_{j}-\lambda_{1}\right) & & \\
& & & \\
& & & \\
& & \left(\lambda_{j}-\lambda_{1}\right) & 1 \\
& & \left(\lambda_{j}-\lambda_{1}\right)
\end{array}\right) \quad\left(l_{j}=1,2, \cdots, i_{j}\right)
\end{aligned}
$$

where $j=2, \cdots, k$.

Because $z_{l_{1}}^{1} \leq n_{1} \quad\left(l_{1}=1,2, \cdots, i_{1}\right)$,through calculation , we have that $\left(E_{z_{l_{1}}^{1}}^{*}\right)^{n_{1}}=O_{z_{l_{1}}^{1}}$ where

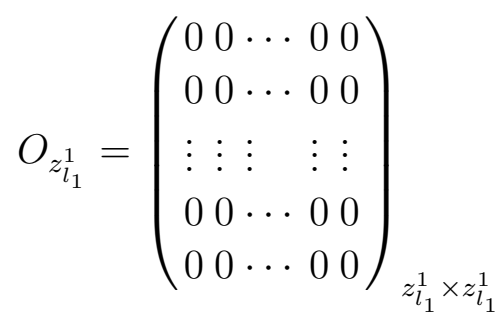

Because $\bar{E}_{z_{l_{j}}^{j}},(j=2,3, \cdots, k)$ are upper triangular matrix with diagonal element $\left(\lambda_{j}-\lambda_{1}\right)^{n_{1}}, \bar{E}_{z_{l_{j}}}$ is invertible. Appreciably, the set

$$
\begin{gathered}
e_{1}=\underbrace{(\overbrace{1,0,0, \cdots, 0}^{n_{1}}, 0, \cdots, 0)}_{n}, e_{2}=\underbrace{(\overbrace{0,1,0, \cdots, 0}^{n_{1}}, 0, \cdots, 0)}_{n}, \cdots, \\
e_{n_{1}}=\underbrace{(\overbrace{0,0,0, \cdots, 1}^{n_{1}}, 0, \cdots, 0)}_{n}
\end{gathered}
$$

is a basis of the solution space $\bar{U}_{1}$ of $(2.6)$, then any basis $\bar{v}_{1}^{1}, \bar{v}_{2}^{1}, \cdots, \bar{v}_{n_{1}}^{1}$ of the solution space $\bar{U}_{1}$ of linear equations (2.6) can be linear represented by 
$e_{1}, e_{2}, \cdots, e_{n_{1}}$ : that is

$$
\begin{aligned}
& \bar{v}_{1}^{1}=\underbrace{(\overbrace{a_{1}^{1}, a_{2}^{1}, a_{3}^{1}, \cdots, a_{n_{1}}^{1}}^{n_{1}}, 0, \cdots, 0)}_{n}, \quad \bar{v}_{2}^{1}=\underbrace{(\overbrace{a_{1}^{2}, a_{2}^{2}, a_{3}^{2}, \cdots, a_{n_{1}}^{2}}^{n_{1}}, 0, \cdots, 0)}_{n}, \ldots, \\
& \bar{v}_{n_{1}}^{1}=\underbrace{(\overbrace{a_{1}^{n_{1}}, a_{2}^{n_{1}}, a_{3}^{n_{1}}, \cdots, a_{n_{1}}^{n_{1}}}^{n_{1}}, 0, \cdots, 0)}_{n}
\end{aligned}
$$

$a_{l}^{j}\left(j, l=1, \cdots, n_{1}\right)$ are constants. Appreciably $U_{1}=B^{\dashv} \bar{U}_{1}$. Let

$$
v_{1}^{1}=B^{\dashv} \bar{v}_{1}^{1}, v_{2}^{1}=B^{\dashv} \bar{v}_{2}^{1}, \cdots, v_{n_{1}}^{1}=B^{\dashv} \bar{v}_{n_{1}}^{1}
$$

$v_{1}^{1}, v_{2}^{1}, \cdots, v_{n_{1}}^{1}$ is a basis of $U_{1}$.

Taking $\lambda_{j}=\lambda_{2}$ in(2.4) and Let $B u=u^{*}$, a basis of $\bar{U}_{2}$ is that

$$
\begin{aligned}
& e_{n_{1}+1}=\underbrace{(\overbrace{0,0, \cdots, 0}^{n_{1}}, \overbrace{1,0, \cdots, 0}^{n_{2}}, 0, \cdots, 0)}_{n}, e_{n_{1}+2}=\underbrace{(\overbrace{0,0, \cdots, 0}^{n_{n}}, \overbrace{0,1, \cdots, 0}^{n_{1}}, 0, \cdots, 0)}_{n}, \ldots, \\
& e_{n_{1}+n_{2}}=\underbrace{\overbrace{0,0, \cdots, 0}^{n_{1}}, \overbrace{0,0, \cdots, 1}^{n_{2}}, 0, \cdots, 0)}_{n}
\end{aligned}
$$

Correspond to $\lambda_{2}$, a basis of the solution space of $\bar{U}_{2}$ :

$$
\bar{v}_{1}^{2}, \bar{v}_{2}^{2}, \cdots, \bar{v}_{n_{2}}^{2}
$$

can be written as

$$
\begin{aligned}
& \bar{v}_{1}^{2}=\underbrace{(\overbrace{0,0, \cdots, 0}^{n_{1}}, \overbrace{b_{1}^{1}, b_{2}^{1}, \cdots, b_{n_{2}}^{1}}^{n_{2}}, 0, \cdots, 0)}_{n}, \quad \bar{v}_{2}^{2}=\underbrace{(\overbrace{0,0, \cdots, 0}^{n_{1}}, \overbrace{b_{1}^{2}, b_{2}^{2}, \cdots, b_{n_{2}}^{2}}^{n_{2}}, 0, \cdots, 0)}_{n}, \ldots, \\
& \bar{v}_{n_{2}}^{2}=\underbrace{\overbrace{0,0, \cdots, 0}^{n_{1}}, \overbrace{b_{1}^{n_{2}}, b_{2}^{n_{2}}, \cdots, b_{n_{2}}^{n_{2}}}^{n_{2}}, 0, \cdots, 0)}_{n}
\end{aligned}
$$

$b_{l}^{j}\left(j, l=1, \cdots, n_{2}\right)$ are constants. Appreciably $U_{2}=B^{\dashv} \bar{U}_{2}$. Let

$$
v_{1}^{2}=B^{\dashv} \bar{v}_{1}^{2}, v_{2}^{2}=B^{\dashv} \bar{v}_{2}^{2}, \cdots, v_{n_{2}}^{2}=B^{\dashv} \bar{v}_{n_{2}}^{2}
$$

then $v_{1}^{2}, v_{2}^{2}, \cdots, v_{n_{2}}^{2}$ is a basis of $U_{2}$.

By a similar argument as above, we obtain a basis of subspace $\bar{U}_{k}$ about $\lambda_{k}$ :

$$
\bar{v}_{1}^{k}, \bar{v}_{2}^{k}, \cdots, \bar{v}_{n_{k}}^{k}
$$


They can be represented as the following:

$$
\begin{aligned}
& \bar{v}_{1}^{k}=\underbrace{(0, \cdots, 0, \overbrace{d_{1}^{1}, d_{2}^{1}, \cdots, d_{n_{k}}^{1}}^{n_{k}})}_{n}, \quad \bar{v}_{2}^{k}=\underbrace{(0, \cdots, 0, \overbrace{d_{1}^{2}, d_{2}^{2}, \cdots, d_{n_{k}}^{2}}^{n_{k}})}_{n}, \ldots, \\
& \bar{v}_{n_{k}}^{k}=\underbrace{(0, \cdots, 0, \overbrace{d_{1}^{n_{k}}, d_{2}^{n_{k}}, \cdots, d_{n_{k}}^{n_{k}}}^{n_{k}})}_{n}
\end{aligned}
$$

$d_{l}^{j}\left(j, l=1, \cdots, n_{k}\right)$ are constants. Appreciably $U_{k}=B^{\dashv} \bar{U}_{k}$, Let

$$
v_{1}^{k}=B^{\dashv} \bar{v}_{1}^{k}, v_{2}^{k}=B^{\dashv} \bar{v}_{2}^{k}, \cdots, v_{n_{k}}^{k}=B^{\dashv} \bar{v}_{n_{k}}^{k}
$$

$v_{1}^{k}, v_{2}^{k}, \cdots, v_{n_{k}}^{k}$ is a basis of $U_{k}$.

Appreciably $\bar{v}_{1}^{1}, \bar{v}_{2}^{1}, \cdots, \bar{v}_{n_{1}}^{1}, \bar{v}_{1}^{2}, \bar{v}_{2}^{2}, \cdots, \bar{v}_{n_{2}}^{2}, \cdots, \bar{v}_{1}^{k}, \bar{v}_{2}^{k}, \cdots, \bar{v}_{n_{k}}^{k}$ are linear independence. because

$$
n_{1}+n_{2}+\cdots+n_{k}=n
$$

then

$$
\bar{v}_{1}^{1}, \bar{v}_{2}^{1}, \cdots, \bar{v}_{n_{1}}^{1}, \bar{v}_{1}^{2}, \bar{v}_{2}^{2}, \cdots, \bar{v}_{n_{2}}^{2}, \cdots, \bar{v}_{1}^{k}, \bar{v}_{2}^{k}, \cdots, \bar{v}_{n_{k}}^{k}
$$

is a basis of $\mathrm{n}$ dimension vector space $U$.

$$
v_{1}^{1}, v_{2}^{1}, \cdots, v_{n_{1}}^{1}, v_{1}^{2}, v_{2}^{2}, \cdots, v_{n_{2}}^{2}, \cdots, v_{1}^{k}, v_{2}^{k}, \cdots, v_{n_{k}}^{k}
$$

and

$$
\bar{v}_{1}^{1}, \bar{v}_{2}^{1}, \cdots, \bar{v}_{n_{1}}^{1}, \bar{v}_{1}^{2}, \bar{v}_{2}^{2}, \cdots, \bar{v}_{n_{2}}^{2}, \cdots, \bar{v}_{1}^{k}, \bar{v}_{2}^{k}, \cdots, \bar{v}_{n_{k}}^{k}
$$

are equivalent, so

$$
v_{1}^{1}, v_{2}^{1}, \cdots, v_{n_{1}}^{1}, v_{1}^{2}, v_{2}^{2}, \cdots, v_{n_{2}}^{2}, \cdots, v_{1}^{k}, v_{2}^{k}, \cdots, v_{n_{k}}^{k}
$$

is also a basis of $\mathrm{n}$ dimension space $U$. The proof is complete.

If $A$ is $n \times n$ constant matrix, we define matrix exponent $\exp A$ by the following matrix series

$$
\exp A=\sum_{k=0}^{\infty} \frac{A^{k}}{k !}=E+A+\frac{A^{2}}{2 !}+\cdots+\frac{A^{m}}{m !}+\cdots
$$

Here $E$ is $n \times n$ identity matrix, $A^{m}$ is power of degree $m$ of $A$. Where we let $A^{0}=E, 0 !=1$.

$\exp A$ has the following properties:

(1) if matrix $A, B$ is commutative, that is $A B=B A$, then

$$
\exp (A+B)=(\exp A)(\exp B)
$$


(2) if $A$ is a diagonal matrix, that is

$$
A=\left(\begin{array}{cccc}
a_{1} & & & \\
& a_{2} & & \\
& & \ddots & \\
& & & a_{n}
\end{array}\right)
$$

then

$$
\exp A=\left(\begin{array}{llll}
e^{a_{1}} & & & \\
& e^{a_{2}} & & \\
& & \ddots & \\
& & & e^{a_{n}}
\end{array}\right)
$$

Consider the following initial value problem

$$
\left\{\begin{array}{l}
\frac{d x}{d t}=A x \\
x(0)=\eta
\end{array}\right.
$$

where $\eta$ is $\mathrm{n}$ dimension vector.

Theorem 2.2 The solution of the problem (2.7) is that

$$
x(t)=(\exp A t) \eta
$$

The proof of properties of $\exp A$ and theorem(2.2) see ([1]).

\section{Main result}

In this section, we use the same symbol as in section 2 and they represent the same significance as in section 2 .

New we give out the step of solving problem (2.7).

(1) Find $\mathrm{k}$ distinct eigenvalues of $A$ :

$$
\lambda_{1}, \lambda_{2}, \cdots, \lambda_{k}
$$

Their multiplicities are $n_{1}, n_{2}, \cdots, n_{k}$ respectively, bring $\lambda_{j}(j=1,2, \cdots, k)$ into (2.1), find a basis of (2.1), that is: a basis of the solution subspace $U_{j}$ of (2.1) with $\lambda_{j}$ :

$$
v_{1}^{j}, v_{2}^{j}, \cdots, v_{n_{j}}^{j}(j=1,2, \ldots, k)
$$

By theorem $(2.1)$,

$$
v_{1}^{1}, v_{2}^{1}, \cdots, v_{n_{1}}^{1}, v_{1}^{2}, v_{2}^{2}, \cdots, v_{n_{2}}^{2}, \cdots, v_{1}^{k}, v_{2}^{k}, \cdots, v_{n_{k}}^{k}
$$

is a basis of $n$ dimension space.

(2) $\eta$ in (2.7) can be linear represented by the basis of $n$ dimension space:

$$
v_{1}^{1}, v_{2}^{1}, \cdots, v_{n_{1}}^{1}, v_{1}^{2}, v_{2}^{2}, \cdots, v_{n_{2}}^{2}, \cdots, v_{1}^{k}, v_{2}^{k}, \cdots, v_{n_{k}}^{k}
$$


that is to said,the following linear equations:

$$
\begin{aligned}
& \eta=x_{1}^{1} v_{1}^{1}+x_{2}^{1} v_{2}^{1}+\cdots+x_{n_{1}}^{1} v_{n_{1}}^{1}+x_{1}^{2} v_{1}^{2}+x_{2}^{2} v_{2}^{2}+\cdots+x_{n_{2}}^{2} v_{n_{2}}^{2}+\cdots \\
& \quad+x_{1}^{k} v_{1}^{k}+x_{2}^{k} v_{2}^{k}+\cdots+x_{n_{k}}^{k} v_{n_{k}}^{k}
\end{aligned}
$$

are solvable and have unique solution, so we obtain that

$$
v_{j}=x_{1}^{j} v_{1}^{j}+x_{2}^{j} v_{2}^{j}+\cdots+x_{n_{j}}^{j} v_{n_{j}}^{j} \in U_{j} \quad(j=1,2, \ldots, k)
$$

then

$$
\eta=\sum_{j=1}^{k} v_{j}
$$

(3) By theorem(2.2), the solution of (2.7) is that

$$
x(t)=(\exp A t) \eta
$$

Bring (3.2) into above, we have that

$$
x(t)=\sum_{j=1}^{k}(\exp A t) v_{j} .
$$

New we compute $(\exp A t) v_{j}$. By the property of $\exp A$

$$
\begin{aligned}
(\exp A t) v_{j} & =(\exp A t) E v_{j}=(\exp A t) e^{\lambda_{j} t}\left[\exp \left(-\lambda_{j} E t\right)\right] v_{j} \\
& =e^{\lambda_{j} t}\left[\exp \left(A-\lambda_{j} E\right) t\right] v_{j} \\
& =e^{\lambda_{j} t}\left[E+t\left(A-\lambda_{j} E\right)+\frac{t^{2}}{2 !}\left(A-\lambda_{j} E\right)^{2}+\cdots\right. \\
& \left.+\frac{t^{n_{j}-1}}{\left(n_{j}-1\right) !}\left(A-\lambda_{j} E\right)^{n_{j}-1}\right] v_{j}
\end{aligned}
$$

Here we use the following result: when $v_{j} \in U_{j}$, if $i \geq n_{j}$, then

$$
\left(A-\lambda_{j} E\right)^{i} v_{j}=0
$$

Finally, we obtain the expression of the solution

$$
x(t)=\sum_{j=1}^{k} e^{\lambda_{j} t}\left[\sum_{i=0}^{n_{j}-1} \frac{t^{i}}{i !}\left(A-\lambda_{j} E\right)^{i}\right] v_{j}
$$

\section{Application}

EXAMPLE 1 Consider the following problem

$$
\left\{\frac{d x}{d t}=A x\right.
$$

$A$ is $n \times n$ constant matrix. Every solution of the problem (4.1) can be written in the form

$$
x(t)=(\exp A t) \eta
$$


$\eta$ is $\mathrm{n}$ dimension vector. The fundamental matrix of the problem (4.1) expAt can be obtained from solving $\mathrm{n}$ initial problem.

$$
\begin{gathered}
\text { Let } e_{1}=\left[\begin{array}{c}
1 \\
0 \\
\vdots \\
0 \\
\vdots \\
0
\end{array}\right], e_{2}=\left[\begin{array}{c}
0 \\
1 \\
\vdots \\
0 \\
\vdots \\
0
\end{array}\right], \cdots, e_{n}=\left[\begin{array}{c}
0 \\
0 \\
\vdots \\
0 \\
\vdots \\
1
\end{array}\right] \text {, then } \\
\exp A t=(\exp A t) E=\left[(\exp A t) e_{1},(\exp A t) e_{2}, \cdots,(\exp A t) e_{n}\right]
\end{gathered}
$$

$(\exp A t) e_{k}$ is the solution of the following problem

$$
\left\{\begin{array}{l}
\frac{d x}{d t}=A x \\
x(0)=e_{k}
\end{array} \quad(k=1, \ldots, n)\right.
$$

Obviously,(4.3) is solvable. So we have obtained expAt.

EXAMPLE 2 Considered the following systems of differential equations

$$
\begin{gathered}
\left\{\begin{array}{c}
x_{1}^{\prime}=x_{1}+2 x_{2}+x_{3} \\
x_{2}^{\prime}=x_{1}-x_{2}+x_{3} \\
x_{3}^{\prime}=2 x_{1}+x_{3}
\end{array}\right. \\
x(0)=\left[\begin{array}{l}
1 \\
0 \\
0
\end{array}\right]
\end{gathered}
$$

Where the coefficient matrix is that

$$
A=\left(\begin{array}{ccc}
1 & 2 & 1 \\
1 & -1 & 1 \\
2 & 0 & 1
\end{array}\right)
$$

SOLUTION Characteristic equation of $\mathrm{A}$ is

$$
\operatorname{det}(A-\lambda E)=(\lambda+1)^{2}(\lambda-3)=0
$$

$\lambda_{1}=3, \lambda_{2}=-1$ are $n_{1}=1, n_{2}=2$ multiplicity eigenvalue respectively. The subspace $U_{1}, U_{2}$ that are correspond to $\lambda_{1}, \lambda_{2}$ are spaces of solutions of $(A-3 E) u=0,(A+E)^{2} u=0$ respectively.

First, we discuss that

$$
(A-3 E) u=\left(\begin{array}{ccc}
-2 & 2 & 1 \\
1 & -4 & 1 \\
2 & 0 & -2
\end{array}\right) u=0
$$


or

$$
\left\{\begin{array}{l}
-2 u_{1}+2 u_{2}+u_{3}=0 \\
u_{1}-4 u_{2}+u_{3}=0 \\
2 u_{1}-2 u_{3}=0
\end{array}\right.
$$

A basis of solution space of linear equations is that

$$
v_{1}^{1}=\left[\begin{array}{l}
-1 \\
-\frac{1}{2} \\
-1
\end{array}\right]
$$

Next, we discuss that

$$
(A+E)^{2} u=\left(\begin{array}{lll}
8 & 4 & 6 \\
4 & 2 & 3 \\
8 & 4 & 6
\end{array}\right) u=0
$$

or

$$
\left\{\begin{array}{l}
8 u_{1}+4 u_{2}+6 u_{3}=0 \\
4 u_{1}+2 u_{2}+3 u_{3}=0 \\
8 u_{1}+4 u_{2}+6 u_{3}=0
\end{array}\right.
$$

A basis of space of solution is

$$
v_{1}^{2}=\left[\begin{array}{c}
-\frac{1}{2} \\
1 \\
0
\end{array}\right] \quad v_{2}^{2}=\left[\begin{array}{c}
-\frac{3}{4} \\
0 \\
1
\end{array}\right]
$$

then $v_{1}^{1}, v_{1}^{2}, v_{2}^{2}$ is a basis of three dimension vector space. Solving the following linear equations

$$
\left[\begin{array}{l}
1 \\
0 \\
0
\end{array}\right]=\alpha_{1}\left[\begin{array}{l}
-1 \\
-\frac{1}{2} \\
-1
\end{array}\right]+\alpha_{2}\left[\begin{array}{c}
-\frac{1}{2} \\
1 \\
0
\end{array}\right]+\alpha_{3}\left[\begin{array}{c}
-\frac{3}{4} \\
0 \\
1
\end{array}\right]
$$

We have that $\alpha_{1}=-\frac{1}{2}, \alpha_{2}=-\frac{1}{4}, \alpha_{3}=-\frac{1}{2}$, then

$$
v_{1}=\left[\begin{array}{c}
\frac{1}{2} \\
\frac{1}{4} \\
\frac{1}{2}
\end{array}\right] \quad v_{2}=\left[\begin{array}{c}
\frac{1}{2} \\
-\frac{1}{4} \\
-\frac{1}{2}
\end{array}\right]
$$

We obtain the solution of the initial problem

$$
x(t)=e^{3 t} E v_{1}+e^{-t}(E+t(A+E)) v_{2}
$$

that is

$$
x(t)=e^{3 t}\left[\begin{array}{c}
\frac{1}{2} \\
\frac{1}{4} \\
\frac{1}{2}
\end{array}\right]+e^{-t}\left[\begin{array}{c}
\frac{1}{2} \\
-\frac{1}{4} \\
-\frac{1}{2}
\end{array}\right]
$$


EXAMPLE 3 We considered the following systems of differential equations

$$
\begin{gathered}
\left\{\begin{array}{l}
x_{1}^{\prime}=3 x_{1}-x_{2}+x_{3} \\
x_{2}^{\prime}=2 x_{1}+x_{3} \\
x_{3}^{\prime}=x_{1}-x_{2}+2 x_{3}
\end{array}\right. \\
x(0)=\left[\begin{array}{l}
\eta_{1} \\
\eta_{2} \\
\eta_{3}
\end{array}\right]
\end{gathered}
$$

Where the coefficient matrix is that

$$
A=\left(\begin{array}{ccc}
3 & -1 & 1 \\
2 & 0 & 1 \\
1 & -1 & 2
\end{array}\right)
$$

SOLUTION Characteristic equation of $\mathrm{A}$ is

$$
\operatorname{det}(A-\lambda E)=(\lambda-1)(\lambda-2)^{2}=0
$$

$\lambda_{1}=1, \lambda_{2}=2$ are $n_{1}=1, n_{2}=2$ multiplicity eigenvalue respectively. The subspace $U_{1}, U_{2}$ that are correspond to $\lambda_{1}, \lambda_{2}$ are spaces of solutions of $(A-E) u=0,(A-2 E)^{2} u=0$ respectively.

First we discuss

$$
(A-E) u=\left(\begin{array}{lll}
2 & -1 & 1 \\
2 & -1 & 1 \\
1 & -1 & 1
\end{array}\right) u=0
$$

or

$$
\left\{\begin{array}{l}
2 u_{1}-u_{2}+u_{3}=0 \\
2 u_{1}-u_{2}+u_{3}=0 \\
u_{1}-u_{2}+u_{3}=0
\end{array}\right.
$$

A basis of solution space of systems of linear equations is that

$$
v_{1}^{1}=\left[\begin{array}{l}
0 \\
1 \\
1
\end{array}\right]
$$

Next, we discuss

$$
(A-2 E)^{2} u=\left(\begin{array}{ccc}
0 & 0 & 0 \\
-1 & 1 & 0 \\
-1 & 1 & 0
\end{array}\right) u=0
$$

or

$$
\left\{\begin{array}{l}
-u_{1}+u_{2}=0 \\
-u_{1}+u_{2}=0
\end{array}\right.
$$


A basis of space of solution is

$$
v_{1}^{2}=\left[\begin{array}{l}
0 \\
0 \\
1
\end{array}\right] \quad v_{2}^{2}=\left[\begin{array}{l}
1 \\
1 \\
0
\end{array}\right]
$$

then $v_{1}^{1}, v_{1}^{2}, v_{2}^{2}$ is a basis of three dimension vector space. Solving the following systems of linear equations

$$
\left[\begin{array}{l}
\eta_{1} \\
\eta_{2} \\
\eta_{3}
\end{array}\right]=\alpha\left[\begin{array}{l}
0 \\
1 \\
1
\end{array}\right]+\beta\left[\begin{array}{l}
1 \\
1 \\
0
\end{array}\right]+\gamma\left[\begin{array}{l}
0 \\
0 \\
1
\end{array}\right]
$$

We have that $\alpha=\eta_{2}-\eta_{1}, \beta=\eta_{1}, \gamma=\eta_{3}-\eta_{2}+\eta_{1}$, then

$$
v_{1}=\left[\begin{array}{c}
0 \\
\eta_{2}-\eta_{1} \\
\eta_{2}-\eta_{1}
\end{array}\right] \quad v_{2}=\left[\begin{array}{c}
\eta_{1} \\
\eta_{1} \\
\eta_{3}-\eta_{2}+\eta_{1}
\end{array}\right]
$$

We obtain the solution of the initial problem

$$
x(t)=e^{t} E v_{1}+e^{2 t}(E+t(A-2 E)) v_{2}
$$

that is

$$
x(t)=e^{t}\left[\begin{array}{c}
0 \\
\eta_{2}-\eta_{1} \\
\eta_{2}-\eta_{1}
\end{array}\right]+e^{2 t}\left[\begin{array}{c}
\eta_{1}+t\left(\eta_{3}-\eta_{2}+\eta_{1}\right) \\
\eta_{1}+t\left(\eta_{3}-\eta_{2}+\eta_{1}\right) \\
\eta_{3}-\eta_{2}+\eta_{1}
\end{array}\right]
$$

\section{REFERENCES}

[1] W. Walter, Ordinary Differential Equations, Springer-verlag, 1998.

[2] V.I.Arnol'd, Ordinary Differential Equations, Springer-verlag, 1992.

[3] G.Williams, Linear Algebra with Applications, Jones And Barltett, 2005.

[4] J.B.Fraleigh and R.A.Beauregard, Linear Algebra, Addison-Wesley, 1995.

\section{Received: May 13, 2007}

